\title{
Editorial
}

\section{Robots are Leaping From the Factory into the Operation Theatre ....}

\author{
M M Haque ${ }^{\top}$
}

The practice of general surgery has been dramatically revolutionized and innovated by the advent of endoscopic microsurgery. Following the initial reports of first successful laparoscopic chloecystectomy in 1987. there has been in explosive proliferation of instrumental technology in the field of Minimal Access Surgery (MAS). Almost every organ is now amenable to endoscopic approach. Although many of the specialists consider Japaroscopic surgery as the defining event in the growth of MAS, the real fruits of MAS are yet to be haryested.

Robots are participating in the surgical interventions for some very good reasons. The robots do follow orders, do not cough or sneeze, do not faint at the sight of blood. and most importantly they are masters of precision. The engineers arid surgeons are linking robots to computer work slation and imaging equipment, Using these tools, scientists can see inside the body and create computer models.

Computer-entanced surgery is being done now and will continue to be evolved rapidly in the future. Computers will eventually participate in surgery actively through robots. These robots will not replace surgeons, but will serve the surgeons by doing tedious tasks tike holding the camera, retracting the viscera and repetitions of few tedious jobs and so on. And thus enhancing the dexierity of the surgeon. It is a sort of master slave manipulator under immediate and constant control of the surgeon.'
With computer-enlanced surgery the surgeon need no longer be in the direct contact with the patient. The surgeon's action can be transmilted through a robotic interface that can eliminate tremor and scale motions. It is believed that such computerenhanced surgery will allow greater precision than is currently possible to achieve by hands. Among the robotic systems currently in use are a robotic arm used for holding a laparoscope or grasping instruments, and a robotic device used to precisety drill out femur for replacement of a hip prosthesis. This system has been also validated in the performance of delicate procedures such as fallopian tube re-anastomosis or coronary artery bypass surgery and so on. Although these procedures have been done with the surgeon near the patient, the interposition of a robotic interface could enable the surgeon to work at a great distance from the patient- i.e. by telepresence surgery. The theory of all of this is that once the accurate technology is achieved surgeons in the USA will be able to operate on a patien in Bangladesh. The progress depends on continued advances in 3-D imaging and on how to manaye time. Even with command travelling at the speed of light, there can be one second lapses between is surgeon and a faraway robot.

With all the advantages, robots have got their own limitations. The human arm has seven degrees of movement which allow it to place the hand in any arbitrary position. The hands are equipped with a vast array of tactile proprioceptive receptors with

'Associate Prolassce. Department of Surgery. Aaşhahi Mececal College, Rajsuahi. 
automatic feedback neural loops. In contrast only four degrees of freedom are available with the courrent generation of instruments used in the robotic surgery ${ }^{2}$ The component activity of surgical dissection is unpredictable and varies substantially between patients undergoing the same operation. It is very much difficult to program the robots 10 all the unpredictable surgical realities. In any case robotics will enhance surgeon's dexterity and extend the ability to deliver care in remote locations.

In the light of anticipated changes our conventional training methods in surgery require a gradual but rapid reappraisal. The next generation of surgeons will be changes in our professional direction. If we do not demand the changes for ourselves, our patients definitely will.

\section{References}

1. Cuschieri A, Steele R.JC. Moossa AR. Minimal Access Therapy. In: Essentials of Surgical PracticeBasic Surgical Training: London. Amold 2001.p. 493-519.

2. Cuschieri A, Giles GR, Moossa AR. Minimal Access Surgery, In: Essential Surgical Practice. London Butterworth- Heinemann Ltd. 1995, p. 1451-62.

All correspondence to: M Manzurul Haque Associale Prolessor Department of Surgery Rajshahi Medical College Resshahi 